Journal of Applied Mathematics and Stochastic Analysis, 16:4 (2003), 349-360.

Printed in the USA (C)2003 by North Atlantic Science Publishing Company

\title{
A SPECTRAL APPROACH TO COMPUTE THE MEAN PERFORMANCE MEASURES OF THE QUEUE WITH LOW-ORDER BMAP INPUT ${ }^{1}$
}

\author{
HO WOO LEE \\ Sung Kyun Kwan University \\ Dept. of Systems Management Engineering \\ Su Won, Korea 440-746 \\ E-mail:hwlee@yurim.skku.ac.kr \\ JONG MIN MOON \\ SE Application Prog. Info Tech. Group Division LG CNS \\ Good Morning Bldg. Yeo Eui Do \\ Seoul 150-712, Korea \\ E-mail:dbmap@bcline.com \\ JONG KEUN PARK \\ I/O System Team, Computer System Department \\ Computer \& Software Research Laboratory, ETRI \\ Dae Jon 305-350, Korea \\ E-mail: queue@etri.re.kr \\ and \\ BYUNG KYU KIM \\ Itsweb, CRM Team, RE D Center \\ 789-4 Young Bldg, Yok Sam Dong Kang Nam \\ Seoul 135-080, Korea \\ E-mail: getstar@itsweb.co.kr
}

(Received October, 2002; Revised May, 2003)

\begin{abstract}
This paper targets engineers and practitioners who want a simple procedure to compute the mean performance measures of the Batch Markovian Arrival process (BMAP/G/1) queueing system when the parameter matrices order is very low. We develop a set of system equations and derive the vector generating function of the queue length. Starting from the generating function, we propose a spectral approach that can be understandable to those who have basic knowledge of $\mathrm{M} / \mathrm{G} / 1$ queues and eigenvalue algebra.

Key words: BMAP/G/1 Queue, Spectral Approach.

AMS (MOS) subject classification: 60K25.
\end{abstract}

\footnotetext{
${ }^{1}$ This work was supported by KOSEF through Statistical Research Center for Complex Systems at Seoul National University
} 


\section{Introduction}

The purpose of this paper is to present, to practitioners and engineers, a simple approach to compute the mean performance measures of the queueing system into which customers arrive according to a typical non-renewal arrival process called the BMAP (Batch Markovian Arrival Process). The BMAP, with parameter matrices $\mathbf{D}_{k},(k \geq 0)$, is a Markov process $\{X(t), J(t)\}$ with infinitesimal generator

$$
\mathbf{Q}=\left(\begin{array}{ccccc}
\mathbf{D}_{0} & \mathbf{D}_{1} & \mathbf{D}_{2} & \mathbf{D}_{3} & \Lambda \\
\mathbf{0} & \mathbf{D}_{0} & \mathbf{D}_{1} & \mathbf{D}_{2} & \Lambda \\
\mathbf{0} & \mathbf{0} & \mathbf{D}_{0} & \mathbf{D}_{1} & \Lambda \\
M & M & M & M & O
\end{array}\right)
$$

where $X(t)$ represents the number of arrivals during $(0, t)$ and $J(t)$ represents the phase of the underlying Markov chain (UMC) at $t . \mathbf{D}_{0}$ has negative diagonal elements and non-negative off-diagonal elements, $\left\{\mathbf{D}_{k},(k \geq 1)\right\}$ are non-negative arrival rate matrices, and $\mathbf{D}=\sum_{k=0}^{\infty} \mathbf{D}_{k}$ is an irreducible infinitesimal generator of the UMC. Special BMAP cases include the MAP (Markovian Arrival process); the MMPP (Markov Modulated Poisson process); the IPP (Interrupted Poisson process); the phase-type renewal process; the Poisson process; and various superpositions of these. For the definition and more comprehensive treatment of the BMAP, readers are advised to see Lucantoni [7], Lucantoni et al. [8], and Latouche and Ramaswami [6] .

Well-established theories and computational algorithms exist concerning BMAP/G/1 queues with arbitrary order of parameter matrices. However, there are many cases where real data is fit to one of the BMAP schemes, resulting in order of matrices that are very low for practical purposes (Heffes and Lucantoni [5], Yousef and Schormans [17]). Despite these low-order parameter matrices, it is a formidable task for practitioners, who have completed Gross and Harris [4], Cooper [2], or Takagi [16], to understand published theories and write computer code even for the simplest MAP/G/1 queues. This has motivated our research.

Classical theory on BMAP/G/1 is based on the matrix-analytic method pioneered by Neuts $[9,10]$. The BMAP was first introduced as a versatile Markovian point process in Neuts [11]. The BMAP/G/1 queue was first analyzed by Ramaswami as a N/G/1 queue [15]. Computational algorithms for BMAP/G/1 queues are provided in Lucantoni [7]. Another approach to the BMAP/G/1 queue is spectral analysis based on eigenvalue algebra. Advanced readers are advised to reference Gail, Hantler and Taylor [3], Nishimura [12], Nishimura and Jiang [13], and Nishimura and Sato [14] (and references therein). Although our theoretical background is very similar to those cited above, our approach is more practical because our spectral approach is intended for field practitioners and provides step-by-step procedures to follow.

We initially derive the system equations for queue length by adopting the supplementary variable technique. We then use the eigenvalues and eigenvectors of the matrix generating function $-\mathbf{D}(z)=-\sum_{n=0}^{\infty} z^{n} \mathbf{D}_{n}$ to derive the vector generating function for queue length and mean queue length. 


\section{Analysis}

\subsection{System equations}

Let us define the following notations and probabilities:

$N(t)$ : queue length (number of customers in the system including the one in service) at $t$

$J(t)$ : phase of the underlying Markov chain (UMC) at $t$,

$m$ : dimension of the UMC,

$S(x)$ : distribution function (DF) of the service time,

$s(x)$ : probability density function (pdf) of the service time,

$S^{*}(\theta)$ : Laplace-Stieltjes transform (LST) of $S(x)$,

$S_{R}(t)$ : remaining service time at $t$,

$$
\begin{gathered}
\pi_{i}=\lim _{t \rightarrow \infty} \operatorname{Pr}[J(t)=i], \quad(1 \leq i \leq m), \\
\pi=\left(\pi_{1}, \pi_{2}, \ldots, \pi_{m}\right),
\end{gathered}
$$

e: unit column of size $m$,

$\lambda=\pi \sum_{n=1}^{\infty} n \mathbf{D}_{n} \mathbf{e}:$ mean arrival rate,

$\rho=\lambda E(S)$ : traffic intensity,

$\left(\mathbf{D}_{n}\right)_{i, j} d t$ : probability that during $(t, t+d t]$ an arrival of group size $n$ occurs and the phase of the UMC changes from $i$ to $j$ just after the arrival, under the condition that UMC was in phase $i$ at $t$,

$q_{i}=\operatorname{Pr}[$ server is idle at $t, J(t)=i], \quad(1 \leq i \leq m)$,

$q_{i}=\lim _{\rightarrow t} q_{i}(t)$

$p_{n, i}(x, t) d x=\operatorname{Pr}\left[\right.$ server is busy at $\left.t, N(t)=n, J(t)=i, S_{R}(t) \in(x, x+d x]\right], \quad(n \geq$ 1),

$p_{n, j}=\lim _{t \rightarrow \infty} p_{n, j}(x, t)$.

Denoting $\left(\mathbf{D}_{n}\right)_{i, j}$ as the $(i, j)$ element of the matrix $\mathbf{D}_{n}$, it is easy to derive the following infinitesimal system equation for $q(t)$ :

$$
\begin{gathered}
q_{i}(t+d t)=q_{i}(t)\left[1+\left(\mathbf{D}_{0}\right)_{i, i} d t\right]+\sum_{\substack{j=1 \\
(j \neq i}}^{m} q_{j}(t)\left(\mathbf{D}_{0}\right)_{j, i} d t \\
+p_{1, i}(0, t) d t+o(d t), \quad(1 \leq i \leq m),
\end{gathered}
$$

where $1+\left(\mathbf{D}_{0}\right)_{i, i} d t$ is the probability that no changes occur during $(t, t+d t]$ in both the queue length and the phase of the UMC. Also we get

$$
p_{1, i}(x-d t, t+d t)=p_{1, i}(x, t)\left[1+\left(\mathbf{D}_{0}\right)_{i, i} d t\right]+\sum_{\substack{j=1 \\ j \neq i}}^{m} p_{1, j}(x, t)\left(\mathbf{D}_{0}\right)_{j, i} d t
$$




$$
\begin{gathered}
+p_{2, i}(0, t) s(x) d t+\sum_{j=1}^{m} q_{j}(t)\left(\mathbf{D}_{1}\right)_{j, i} s(x) d t+o(d t),(1 \leq i \leq m), \\
p_{n, i}(x-d t, t+d t)=p_{n, i}(x, t)\left[1+\left(\mathbf{D}_{0}\right)_{i, i} d t\right]+\sum_{\substack{j=1 \\
j \neq i}}^{m} p_{n, j}(x, t)\left(\mathbf{D}_{0}\right)_{j, i} d t \\
+p_{n+1, i}(0, t) s(x) d t+\sum_{j=1}^{m} q_{j}(t)\left(\mathbf{D}_{n}\right)_{j, i} s(x) d t \\
+\sum_{k=1}^{n-1} p_{k, i}(x, t)\left(\mathbf{D}_{n-k}\right)_{i, i} d t \\
+\sum_{k=1}^{n-1} \sum_{\substack{j=1 \\
j \neq i}}^{m} p_{k, j}(x, t)\left(\mathbf{D}_{n-k}\right)_{j, i} d t+o(d t),(1 \leq i \leq m, n \geq 2) .
\end{gathered}
$$

Taking $t \rightarrow \infty$, and using $\mathbf{q}=\left(q_{1}, \ldots, q_{m}\right)$ and $\mathbf{p}_{n}(x)=\left(p_{n, 1}(x), \ldots, p_{n, m}(x)\right)$, we get the following vector system equations:

$$
\begin{gathered}
-\mathbf{q} \mathbf{D}_{0}=\mathbf{p}_{1}(0), \\
-\frac{d}{d x} \mathbf{p}_{n}(x)=\mathbf{q} \mathbf{D}_{n} s(x)+\mathbf{p}_{n+1}(0) s(x)+\sum_{k=1}^{n} \mathbf{p}_{k}(x) \mathbf{D}_{n-k}, \quad(n \geq 1) .
\end{gathered}
$$

Equation (2.1) represents the balance of in-flow and out-flow in UMC phases with zero customers in the system. Equation (2.2) represents the balances in UMC phases when there are $n$ customers in the system.

\subsection{Vector generating function of the queue length}

Let us define the following vector and matrix generating functions,

$$
\mathbf{p}(z, x)=\sum_{n=1}^{\infty} \mathbf{p}_{n}(x) z^{n}, \quad \mathbf{p}(z, 0)=\sum_{n=1}^{\infty} \mathbf{p}_{n}(0) z^{n} .
$$

We multiply equation (2.2) by $z^{n}$, sum over $n=1,2, \ldots$, and use equation (2.1) to get:

$$
-\frac{d}{d x} \mathbf{p}(z, x)=\mathbf{p}(p, x) \mathbf{D}(z)+\left[\frac{1}{z} \mathbf{p}(z, 0)+\mathbf{q} \mathbf{D}(z)\right] s(x) .
$$

Let us define the Laplace transform (LT) $\mathbf{p}^{*}(z, \theta)=\int_{0}^{\infty} \mathbf{p}(z, x) e^{-\theta x} d x$. We take the LT of both sides of equation (2.3) to get

$$
\mathbf{p}^{*}(z, \theta)[\theta \mathbf{I}+\mathbf{D}(z)]=\left[1-\frac{S^{*}(\theta)}{z}\right] \mathbf{p}(z, 0)-\mathbf{q} \mathbf{D}(z) S^{*}(\theta) .
$$

In order for $\mathbf{p}^{*}(z, \theta)$ to be complete, $\mathbf{p}(z, 0)$ must be determined. This can be accomplished by making the left-hand side of equation (2.4) zero. For this purpose, let $\alpha_{1}(z)$, $\alpha_{2}(z), \ldots, \alpha_{m}(z)$ be the eigenvalues of the matrix $\operatorname{GF}-\mathbf{D}(z)=-\sum_{n=0}^{\infty} \mathbf{D}_{n} z^{n}$, and 
$\xi_{i}(z)$ be the right eigenvector of $\alpha_{i}(z)$. If we use the eigenvalue $\alpha_{i}(z)$ in $\theta$ of equation (2.4) and postmultiply both sides by its right eigenvector $\xi_{i}(z)$, we get

$$
\begin{gathered}
\mathbf{p}^{*}\left(z, \alpha_{i}(z)\right)\left[\alpha_{i}(z) \mathbf{I}+\mathbf{D}(z)\right] \xi_{i}(z) \\
=\left\{\left[1-\frac{S^{*}\left(\alpha_{i}(z)\right)}{z}\right] \mathbf{p}(z, 0) \mathbf{q} \mathbf{D}(z) S^{*}\left(\alpha_{i}(z)\right)\right\} \xi_{i}(z) .
\end{gathered}
$$

Because $\alpha_{i}(z)$ and $\xi_{i}(z)$ are related by $-\mathbf{D}(z) \xi_{i}(z)=\alpha_{i}(z) \xi_{i}(z)$, the left-hand side of equation (2.5) vanishes and yields:

$$
\mathbf{p}(z, 0) \xi_{i}(z)=\frac{z \alpha_{i}(z) S^{*}\left(\alpha_{i}(z)\right)}{S^{*}\left(\alpha_{i}(z)\right)-z} \mathbf{q} \xi_{i}(z)
$$

The above identity should hold for any eigenvalue of $-\mathbf{D}(z)$ and its right eigenvector. Thus we have

$$
\left.\mathbf{p}(z, 0)] \xi_{1}(z), \ldots, \xi_{m}(z)\right]=z \mathbf{q}\left[\phi_{1}(z) \xi_{1}(z), \ldots, \phi_{m}(z) \xi_{m}(z)\right]
$$

where $\phi_{i}(z)=\frac{\alpha_{i}(z) S^{*}\left(\alpha_{i}(z)\right)}{S^{*}\left(\alpha_{i}(z)\right)-z}$.

For further analyses, we make the following two assumptions:

Assumption 1: $\mathbf{D}(z)$ is analytic in a neighborhood of $z=1$.

Assumption 2: All eigenvalues of $\mathbf{D}(z)$ are simple for $|z| \leq 1$.

For other analyses of various queueing systems under these assumptions, readers are advised to see Nishimura [12], Nishimura and Jiang [13], and Nishimura and Sato [14].

Under Assumption 2, the inverse matrix $\left[\xi_{1}(z), \xi_{2}(z), \ldots, \xi_{m}(z)\right]^{-1}$ exists. Then, equation (2.7) becomes

$$
\mathbf{p}(z, 0)=z \mathbf{q}\left[\xi_{1}(z), \ldots, \xi_{m}(z)\right] \mathbf{D}_{g}\left\{\phi_{1}(z), \phi_{2}(z), \ldots, \phi_{m}(z)\right\}\left[\xi_{1}(z), \ldots, \xi_{m}(z)\right]^{-1},
$$

where

$$
\mathbf{D}_{g}\left\{x_{1}, x_{2}, \ldots, x_{m}\right\}=\left[\begin{array}{cccc}
x_{1} & 0 & \ldots & 0 \\
0 & x_{2} & \ldots & 0 \\
M & M & \ddots & M \\
0 & 0 & \ldots & x_{m}
\end{array}\right] .
$$

Denoting $\Theta(z)=\left[\xi_{1}(z), \xi_{2}(z), \ldots, x i_{m}(z)\right]$ as the matrix of the eigenvectors, equation (2.7) becomes

$$
\mathbf{p}(z, 0)=z \mathbf{q} \Theta(z) \mathbf{D}_{g}\left\{\phi_{1}(z), \phi_{2}(z), \ldots, \phi_{m}(z)\right\} \Theta^{-1}(z) .
$$

Using equation (2.8) in equation (2.4) yields

$$
\begin{gathered}
\mathbf{p}^{*}(z, \theta)[\theta \mathbf{I}+\mathbf{D}(z)] \\
=\left[z-S^{*}(\theta)\right] \mathbf{q} \Theta(z) \mathbf{D}_{g}\left\{\phi_{1}(z), \phi_{2}(z), \ldots, \phi_{m}(z)\right\} \Theta^{-1}(z)-\mathbf{q} \mathbf{D}(z) S^{*}(\theta) .
\end{gathered}
$$

Using $\theta=0, z=1$, and $\left.\mathbf{D}(z)\right|_{z=1}=\mathbf{D}$ in equation (2.9), we get

$$
\left[\mathbf{p}^{*}(1,0)+\mathbf{q}\right] \mathbf{D}=\mathbf{0} .
$$


Note that the $i$ th element of the vector $\mathbf{p}^{*}(1,0)$ is the joint probability that the server is busy and the phase of the UMC is in $i$. Thus we have $\mathbf{p}^{*}(1,0)+\mathbf{q}=\pi$, which leads to

$$
\pi \mathbf{D}=\mathbf{0}
$$

Equation (2.11) confirms that $\pi$ is the stationary probability vector of the UMC.

Using $\theta=0$ in equation (2.9), we get

$$
\mathbf{p}^{*}(z, 0) \mathbf{D}(z)=(z-1) \mathbf{q} \Theta(z) \mathbf{D}_{g}\left\{\phi_{1}(z), \phi_{2}(z), \ldots, \phi_{m}(z)\right\} \Theta^{-1}(z)-\mathbf{q} \mathbf{D}(z) .
$$

Using the following diagonalizations:

$$
\mathbf{D}(z)=-\Theta(z) \mathbf{D}_{g}\left\{\alpha_{1}(z), \alpha_{2}(z), \ldots, \alpha_{m}(z)\right\} \Theta^{-1}(z)
$$

and

$$
\mathbf{D}^{-1}(z)=-\Theta(z) \mathbf{D}_{g}\left\{1 / \alpha_{1}(z), 1 / \alpha_{2}(z), \ldots, 1 / \alpha_{m}(z)\right\} \Theta^{-1}(z) .
$$

Equation (2.12) becomes

$$
\begin{gathered}
\mathbf{p}^{*}(z, 0)=(z-1) \mathbf{q} \Theta(z) \mathbf{D}_{g}\left\{\phi_{1}(z), \phi_{2}(z), \ldots, \phi_{m}(z)\right\} \Theta^{-1}(z) \mathbf{D}^{-1}(z)-\mathbf{q} \\
=\mathbf{q} \Theta(z) \mathbf{D}_{g}\left\{\gamma_{1}(z), \gamma_{2}(z), \ldots, \gamma_{m}(z)\right\} \Theta^{-1}(z)-\mathbf{q}
\end{gathered}
$$

where

$$
\gamma_{i}(z)=\frac{(1-z) S^{*}\left(\alpha_{i}(z)\right)}{S^{*}\left(\alpha_{i}(z)\right)-z}
$$

Finally, the vector GF $\mathbf{p}(z)$ of the queue length (in most papers, notation $\mathbf{Y}(z)$ is used rather than our $\mathbf{p}(z)$ ) can be obtained by using equation (2.13) in

$$
\mathbf{p}(z)=\mathbf{q}+\mathbf{p}^{*}(z, 0)
$$

we get

$$
\mathbf{p}(z)=\mathbf{q} \Theta(z) \boldsymbol{\Gamma}(z) \Theta^{-1}(z),
$$

where $\boldsymbol{\Gamma}(z)=\mathbf{D}_{g}\left\{\gamma_{1}(z), \gamma_{2}(z), \ldots, \gamma_{m}(z)\right\}$.

The vector $\operatorname{GF} \mathbf{p}_{q}(z)$ of the number of customers in the waiting line (excluding the one in service) becomes

$$
\mathbf{p}_{q}(z)=\mathbf{q}+\frac{\mathbf{p}^{*}(z, 0)}{z}=\mathbf{q} \Theta(z) \mathbf{H}(z) \Theta^{-1}(z)
$$

where $\mathbf{H}(z)=\mathbf{D}_{g}\left\{\eta_{1}(z), \eta_{2}(z), \ldots, \eta_{m}(z)\right\}$ and $\eta_{i}(z)=\frac{(1-z)}{S^{*}\left(\alpha_{i}(z)\right)-z}$.

\subsection{Obtaining the unknown vector}

The vector PGF from equation (2.16) is complete only after the unknown vector $\mathbf{q}=$ $\left(q_{1}, \ldots, q_{m}\right)$ is obtained. Note that $q_{i}$ is the joint probability that the server is idle, and the UMC is in phase $i$ at an arbitrary point of time in steady-state. The vector q can be obtained by solving the $m$ simultaneous equations that result from using the zeros $z_{1}^{*}, \ldots, z_{m}^{*},\left(\left|z_{i}^{*}\right| \leq 1\right)$ of the denominator $\left\{S^{*}\left(\alpha_{i}(z)\right)-z, i=1,2, \ldots, m\right\}$ in the numerator.

Theorem 2.1: $\quad$ There exists, for some $i$, an eigenvalue $\alpha_{i}(z)$ that satisfies $\left.\alpha_{i}(z)\right|_{z=1}=$ $\alpha_{i}(1)=0$. 
Proof: The eigenvalues of $-\mathbf{D}=-\sum_{n=0}^{\infty} \mathbf{D}_{n}=-\left.\mathbf{D}(z)\right|_{z=1}$ are $\alpha_{1}(1), \alpha_{2}(1), \ldots, \alpha_{m}(1)$. Let $\alpha$ be an eigenvalue and $\xi$ be its right eigenvector. We then have $-\mathbf{D} \xi=\alpha \xi$. The row sum of the matrix $-\mathbf{D}$ is zero since it is the infinitesimal generator of the UMC. Thus $\alpha=0$ and $\xi=\mathbf{e}$ satisfies $-\mathbf{D} \xi=\alpha \xi$, which completes the proof.

In the sequel, we will fix $\alpha_{1}(z)$ as the eigenvalue that satisfies $\alpha_{i}(1)=0$. Readers will see that there exists only one such eigenvalue among $\alpha_{1}(z), \ldots, \alpha_{m}(z)$.

Theorem 2.2: Under Assumption 1, we have

(a) $\left[\frac{d}{d z} \alpha_{1}(z)\right]_{z=1}=-\lambda$ and

(b) $\left[\frac{d}{d z} S^{*}\left(\alpha_{1}(z)\right)\right]_{z=1}=\rho$.

Proof: Let $\xi_{1}(z)$ be the right eigenvector of $\alpha_{1}(z)$. We then have the relationship $-\mathbf{D}(z) \xi_{1}(z)=\alpha_{1}(z) \xi_{1}(z)$. Differentiating both sides with respect to $z$, evaluating at $z=1$, and premultiplying by $\pi$, we get (note that $\xi_{1}(1)=\mathbf{e}$ ),

$$
\left[-\pi \frac{d}{d z} \mathbf{D}(z) e-\pi \mathbf{D} \frac{d}{d z} \xi_{1}(z)\right]_{z=1}=\left[\pi \frac{d}{d z} \alpha_{1}(z) \mathbf{e}\right]_{z=1} .
$$

From $\pi \mathbf{D}=\mathbf{0}$ and $\pi \mathbf{e}=1$, the above equation is reduced to

$$
\left[-\pi \frac{d}{d z} \mathbf{D}(z) \mathbf{e}\right]_{z=1}=\left[\frac{d}{d z} \alpha_{1}(z)\right]_{z=1} .
$$

Then $\left[\pi \frac{d}{d z} \mathbf{D}(z) \mathbf{e}\right]_{z=1}=\pi \sum_{n=0}^{\infty} n \mathbf{D}_{n} \mathbf{e}=\lambda$ completes the proof. The result in (b) is a consequence of part (a).

Theorem 2.3: (Neuts [9]:pp 40) If $\mathbf{A}$ is an irreducible matrix with negative diagonal elements and non-negative off-diagonal elements, such that $\mathbf{A e} \leq \mathbf{0}$, then $\mathbf{A}$ has a simple, non-positive eigenvalue $-a(a \geq 0)$ such that for all other eigenvalues $a_{j}$ of $\mathbf{A}$, we have $\operatorname{Re}\left(a_{j}\right)<-a$.

Theorem 2.4: We have $\operatorname{Re}\left(a_{j}(1)\right)>0,(j=2,3, \ldots, m)$.

Proof: $\mathbf{D}(1)=\left.\mathbf{D}(z)\right|_{z=1}=\mathbf{D}$ is the infinitesimal generator of the UMC. Thus the diagonal elements are negative, the off-diagonal elements are nonnegative, and $\mathbf{D e}=\mathbf{0}$. Thus $\mathbf{D}$ is eligible for being $\mathbf{A}$ (from Theorem 2.3). The eigenvalue $\alpha_{1}(1)=0$ of $-\mathbf{D}$ is also an eigenvalue of $\mathbf{D}$. Thus all other eigenvalues of $\mathbf{D}$ have negative real parts. This means that all other eigenvalues of $-\mathbf{D}$ have positive real parts; thereby, completing the proof.

Theorem 2.5: (Bellman [1]) A matrix $\mathbf{A}$ is called stable if the solution of $\frac{d x}{d t}=\mathbf{A} x$, $\mathbf{x}(0)=\mathbf{c}$ approaches zero as $t \rightarrow \infty$, regardless of the value of $\mathbf{c} . A$ necessary and sufficient condition for the stability of $\mathbf{A}$ is that all the eigenvalues of $\mathbf{A}$ have negative real parts.

Theorem 2.6: For $z$ on $\{z:|z| \leq 1\}$, we have $\operatorname{Re}\left(a_{j}(z)\right)>0,(j=2, \ldots, m)$.

Proof: Let $\mathbf{A}_{n}(t)$ be the probability matrix that $n$ customers arrive during $t$ in a BMAP. Let $\mathbf{A}(z, t)=\sum_{n=0}^{\infty} \mathbf{A}_{n}(t) z^{n}$. We then have $\frac{d}{d t} \mathbf{A}(z, t)=\mathbf{A}(z, t) \mathbf{D}(z)$ (Lucantoni et al. [8]), the solution to which is $\mathbf{A}(z, t)=e^{\mathbf{D}(z) t}$. Expressing $e^{\mathbf{D}(z) t}$ in its Jordan form and taking $t \rightarrow \infty$ shows that $\mathbf{D}(z)$ is stable on $\{z:|z| \leq 1\}$. From Theorem 2.3, with $a_{1}(1)=0$, real parts of the eigenvalues of $\mathbf{D}(z)$ are negative on $\{z:|z| \leq 1\}$. From Theorem 2.4, eigenvalues $\alpha_{2}(z), \ldots, \alpha_{m}(z)$ of $-\mathbf{D}(z)$ have positive real parts on $|z| \leq 1$.

From Theorem 2.6, we see that the $\alpha_{i}(z)$ that satisfies $\alpha_{i}(1)=0$ is unique. 
Theorem 2.7: The denominator $S^{*}\left(\alpha_{j}(z)\right)-z$ of $\gamma_{j}(z)$ in equation (2.16) has, for each $j(j=2,3, \ldots, m)$, exactly one zero $z_{i}^{*}$ within the unit circle.

Proof: Let the closed contour $C$ be $C=\{z:|z|=1\}$. Let $f(z)=-z$ and $g(z)=S^{*}\left(\alpha_{j}(z)\right)$. Denote $\alpha_{j}(z)$ as $\alpha_{j}(z)=a_{j}+b_{j} i$, where $a_{j}$ and $b_{j}$ are real and imaginary parts. We note from Theorem 2.6 that $a_{j}>0$ on $C$ for $j=2,3, \ldots, m$. Now, we have $|f(z)|=|-z|=1$ on $C$, and

$$
\begin{aligned}
|g(z)| & =\left|\int_{0}^{\infty} e^{-\alpha_{j}(z) t} d S(t)\right| \leq \int_{0}^{\infty}\left|e^{\alpha_{j}(z) t}\right| d S(t) \\
& =\int_{0}^{\infty}\left|e^{-\left(a_{j}+b_{j} i\right) t}\right| d S(t)<1=|f(z)| .
\end{aligned}
$$

From the well-known Rouche's theorem, $f(z)$ and $f(z)+g(z)$ have the same number of zeros within the unit circle. It is obvious that $f(z)=-z$ has exactly one zero within the unit circle. Thus, $f(z)+g(z)=S^{*}\left(\alpha_{j}(z)\right)-z$ has exactly one zero within the unit circle.

The numerator of equation (2.16) should vanish at the zeros $z_{2}^{*}, z_{3}^{*}, \ldots, z_{m}^{*}$. We derive $(m-1)$ equations that will be used to determine $\mathbf{q}$. One remaining equation can be obtained from the following theorem.

Theorem 2.8: We have

$$
\mathbf{p}(1)=\pi=\mathbf{q} \Theta(1)\left[\begin{array}{cccc}
(1-\rho)^{-1} & 0 & \ldots & 0 \\
0 & 0 & \ldots & 0 \\
\vdots & \vdots & \ddots & \vdots \\
0 & 0 & \ldots & 0
\end{array}\right] \Theta^{-1}(1) .
$$

Proof: From equation (2.18), we get $\left|S^{*}\left(\alpha_{j}(1)\right)\right|<1$. If we use $z=1$ in equation (2.14), we have $\gamma_{i}(z)=0$ for $i=2,3, \ldots, m$. We also have $\gamma_{1}(z)=(1-\rho)^{-1}$ from Theorem 2.2 which completes the proof.

We can obtain the $m$ equations to determine $\mathbf{q}=\left(q_{1}, q_{2}, \ldots, q_{m}\right)$ completely.

\section{$3 \quad$ Mean Queue Length}

The mean queue length can be obtained from equation (2.16) as,

$$
\begin{aligned}
& L=\left[\frac{d}{d z} \mathbf{p}(z) \mathbf{e}\right]_{z=1}=\mathbf{q}\left[\frac{d}{d z} \Theta(1)\right]\left[\begin{array}{cccc}
(1-\rho)^{-1} & 0 & \ldots & 0 \\
0 & 0 & \ldots & 0 \\
\vdots & \vdots & \ddots & \vdots \\
0 & 0 & \ldots & 0
\end{array}\right] \\
& +\mathbf{q} \Theta(1)\left[\frac{d}{d z} \Gamma(1)\right] \Theta^{-1}(1) \mathbf{e}+\mathbf{q} \Theta(1)\left[\begin{array}{cccc}
(1-\rho)^{-1} & 0 & \ldots & 0 \\
0 & 0 & \ldots & 0 \\
\vdots & \vdots & \ddots & \vdots \\
0 & 0 & \ldots & 0
\end{array}\right]\left[\frac{d}{d z} \Theta^{-1}(1)\right] \mathbf{e},
\end{aligned}
$$

where

$$
\frac{d}{d z} \Theta(1)=\left[\frac{d}{d z} \Theta(z)\right]_{z=1}, \frac{d}{d z} \boldsymbol{\Gamma}(1)=\mathbf{D}_{g}\left\{\left[\frac{d}{d z} \gamma_{1}(z)\right]_{z=1}, \ldots,\left[\frac{d}{d z} \gamma_{1}(z)\right]_{z=1}\right\}
$$




$$
\begin{aligned}
& {\left[\frac{d}{d z} \gamma_{1}(z)\right]_{z=1}=\left[\frac{d}{d z}\left(\frac{(1-z) S^{*}\left(\alpha_{1}(z)\right)}{S^{*}\left(\alpha_{1}(z)\right)-z}\right)\right]_{z=1}} \\
& =\frac{\rho}{1-\rho}+\left[\frac{\lambda^{2} E\left(S^{2}\right)-E(S)\left[\frac{d^{2}}{d z^{2}} \alpha_{1}(z)\right]}{2(1-\rho)^{2}}\right]_{z=1},
\end{aligned}
$$

and, for $i=2,3, \ldots, m$,

$$
\begin{aligned}
& {\left[\frac{d}{d z} \gamma_{i}(z)\right]_{z=1}=\left[\frac{d}{d z}\left(\frac{(1-z) S^{*}\left(\alpha_{i}(z)\right)}{S^{*}\left(\alpha_{i}(z)\right)-z}\right)\right]_{z=1} } \\
= & {\left[\frac{S^{*}\left(\alpha_{i}(z)\right)\left[z-S^{*}\left(\alpha_{i}(z)\right)\right]}{\left[S^{*}\left(\alpha_{i}(z)\right)-z\right]^{2}}\right]_{z=1}=\frac{S^{*}\left(\alpha_{i}(1)\right)}{1-S^{*}\left(\alpha_{i}(1)\right)} . }
\end{aligned}
$$

$L_{q}$ can be obtained from

$$
L_{q}=L-\rho .
$$

From the Little's law, we respectively have mean sojourn time $W$ and mean waiting time $W_{q}$ as

$$
W=\frac{L}{\lambda}, \quad W_{1}=\frac{L_{q}}{\lambda} .
$$

\subsection{Summarized steps}

Following summarizes the above procedure that leads to the mean BMAP/G/1 performance measures.

(STEP-1) Obtain $\mathbf{D}(z)$.

(STEP-2) Use $\pi \mathbf{D}=\mathbf{0}, \pi \mathbf{e}=1$ to compute $\pi$. Calculate $\lambda=\pi \sum_{n=1}^{\infty} n \mathbf{D}_{n} \mathbf{e}$ and $\rho=\lambda E(S)$.

(STEP-3) Obtain $m$ eigenvalues $\alpha_{1}(z), \alpha_{2}(z), \ldots, \alpha_{m}(z)$ of $-\mathbf{D}(z)$. Let $\alpha_{1}(z)$ be the one that satisfies $\alpha_{i}(1)=0$ (this is unique).

(STEP-4) Obtain the right eigenvectors $\xi_{1}(z), \ldots, \xi_{m}(z)$ of $\alpha_{1}(z), \ldots, \alpha_{m}(z)$, the matrix $\Theta(z)$ of eigenvectors, and the inverse matrix $\Theta^{-1}(z)$.

(STEP-5) Obtain $\gamma_{i}(z)=\frac{(1-z) S^{*}\left(\alpha_{i}(z)\right)}{S^{*}\left(\alpha_{i}(z)\right)-z}(i=1,2, \ldots, m)$, and use equation $(2.16)$ to get $\mathbf{p}(z)$.

(STEP-6) Obtain equation (2.19).

(STEP-7) Obtain the zero $z_{i}^{*}$ of the denominator $S^{*}\left(\alpha_{i}(z)\right)-z$ of $\gamma_{i}(z)$ that lies within the unit circle for each $i,(i=2,3, \ldots, m)$.

(STEP-8) Evaluate the numerator of equation (2.16) at $z_{2}^{*}, z_{3}^{*}, \ldots, z_{m}^{*}$, and equate the resulting polynomials to zeros. These lead to $m-1$ equations. Together with equation (2.19), we have $m$ equations.

(STEP-9) Solve the $m$ equations to determine $\mathbf{q}$.

(STEP-10) Use equations (3.1)-(3.3) to get the mean performance measures.

\section{$3.2 \quad$ A numerical example}

We consider a BMAP/G/1 queue with parameter matrices $\mathbf{D}_{0}=\left[\begin{array}{cc}-2.5 & 1 \\ 0.4 & -1.5\end{array}\right]$, $\mathbf{D}_{1}=\left[\begin{array}{ll}0.1 & 0.7 \\ 0.3 & 0.2\end{array}\right], \mathbf{D}_{2}=\left[\begin{array}{cc}0.3 & 0.1 \\ 0.1 & 0.2\end{array}\right]$ and $\mathbf{D}_{3}=\left[\begin{array}{ll}0.1 & 0.2 \\ 0.2 & 0.1\end{array}\right]$. We assume that the service time follows an Erlang distributiuon of order 2 with the LT $S^{*}(\theta)=\left(\frac{10}{\theta+10}\right)^{2}$. 
First, we have $\mathbf{D}(z)=\left[\begin{array}{cc}-2.5+0.1 z+0.3 z^{2}+0.1 z^{3} & 1+0.7 z+0.1 z^{2}+0.2 z^{3} \\ 0.4+0.3 z+0.1 z^{2}+0.2 z^{3} & -1.5+0.2 z+0.2 z^{2}+0.1 z^{3}\end{array}\right]$. From $\pi \mathbf{D}=\mathbf{0}, \pi \mathbf{e}=1$, we get $\pi=\left(\frac{1}{3}, \frac{2}{3}\right), \lambda=\pi \sum_{n=1}^{3} n \mathbf{D}_{n} \mathbf{e}=2.166667$, and $\rho=\lambda E(S)$ $=0.433333$. The eigenvalues of $-\mathbf{D}(z)$ are

$$
\alpha_{1}(z)=\frac{4=0.3 z-0.5 z^{2}-0.2 z^{3}-0.4 A}{2}, \alpha_{2}(z)=\frac{4-0.3 z-0.5 z^{2}-0.2 z^{3}+0.4 A}{2},
$$

with

$$
A=\sqrt{\left(2.23313-1.10684 z+z^{2}\right)\left(4.60273-0.318479 z+z^{2}\right)\left(1.58097+2.42532 z+z^{2}\right)} .
$$

Note that $\alpha_{1}(1)=0$. After obtaining the eigenvectors of the eigenvalues, we get

$$
\begin{gathered}
\Theta^{-1}(z)=\frac{1}{\left(1+0.7 z+0.1 z^{2}+0.2 z^{3}\right)\left(\alpha_{1}(z)-\alpha_{2}(z)\right)} \\
\times\left[\begin{array}{cc}
2.5-\alpha_{2}(z)-0.1 z-0.3 z^{2}-0.1 z^{3} & -1-0.7 z-0.1 z^{2}-0.2 z^{3} \\
-2.5+\alpha_{1}(z)+0.1 z+0.3 z^{2}+0.1 z^{3} & 1+0.7 z+0.1 z^{2}+0.2 z^{3}
\end{array}\right] .
\end{gathered}
$$

From $S^{*}(\theta)=\left(\frac{10}{\theta+10}\right)^{2}$, we get $\gamma_{i}(z)=\frac{100(1-z)}{100-z\left(\alpha_{i}(z)+10\right)^{2}}$. Now, we use equation $(2.19)$ to get an equation $q_{1}+q_{2}=0.566667$. We denote the terms of $\mathbf{p}(z)$ of equation $(2.16)$ as $\Theta(z)=\left[\begin{array}{ll}a_{11} & a_{12} \\ a_{21} & a_{22}\end{array}\right], \boldsymbol{\Gamma}(z)=\left[\begin{array}{cc}b_{i 1} & 0 \\ 0 & b_{22}\end{array}\right], d^{-1} \Theta^{-1}(z)=\left[\begin{array}{ll}c_{11} & c_{12} \\ c_{21} & c_{22}\end{array}\right]$ and $d=\left[\left(1+0.7 z+0.1 z^{2}+0.2 z^{3}\right)\left(\alpha_{1}(z)-\alpha_{2}(z)\right)\right]^{-1}$. Consequently, we derive

$$
\begin{gathered}
\mathbf{p}(z)=d\left\{c_{11} b_{11}\left(q_{1} a_{11}+q_{2} a_{21}\right)+c_{21} b_{22}\left(q_{1} a_{12}+q_{2} a_{22}\right),\right. \\
\left.c_{12} b_{11}\left(q_{z} a_{1}+q_{2} a_{21}\right)+c_{22} b_{22}\left(q_{1} a_{12}+q_{2} a_{22}\right)\right\} .
\end{gathered}
$$

The zero of the denominator $100-z\left(\alpha_{2}(z)+10\right)^{2}$ of $b_{22}$ that lies within the unit circle is found to be $z_{2}^{*}=0.59939$, which makes $b_{22}\left(q_{1} a_{12}+q_{2} a_{22}\right)$ zero. We get another equation $1.49857 q_{1}=0.60577 q_{2}$, which produces $\mathbf{q}=\left(q_{1}, q_{2}\right)=(0.163125,0.403542)$.

Finally, from equation (3.1), we get the mean queue length $L=1.129840$. We also get $L_{q}=L-\rho=0.696507, W=0.521465$, and $W_{q}=L_{q} / \lambda=0.321465$ from equations (3.2) and (3.3).

We compared the above mean performance measures with the ones we got from the algorithms of Lucantoni et al. [8], and confirmed that they are equal.

\section{Summary and Discussion}

In this paper, we have presented a simple spectral method to calculate mean performance measures of the queues with a non-renewal inputs. Our motivation was based on the fact that most practitioners who have completed basic queueing text books find it very difficult to understand the theories of the matrix-analytic method and program the published algorithms.

Our method is restrictive in that:

(i) it is based upon the assumption that the eigenvalues of the matrix generating function $\mathbf{D}(z)$ are all distinct, and 
(ii) the eigenvalues of the matrix $\operatorname{GF} \mathbf{D}(z)$ are easy to find.

For most practical problems with low-order parameter matrices, restriction (i) may not pose significant problems. But one may have a problem in finding all the eigenvalues as a function of $z$. In this case, commercially available mathematical software packages may simplify this task.

\section{Acknowledgements}

The first author is very thankful to professor Nishimura of The Science University of Tokyo for helpful comments. Also, professor M.F. Neuts is to be commended for bringing professor Nishimura's works to his attention. The authors are especially thankful for the corrections provided by an anonymous referee which have enhanced the readability of this paper.

\section{References}

[1] Bellman, R., Introduction to Matrix Analysis, Second Edition, Society for Industrial and Applied Mathematics, Philadelphia 1997.

[2] Cooper, R.B., Introduction to Queueing Theory, 2nd ed., North-Holland Pub. Co., New York 1981.

[3] Gail, H.R., Hantler, S.L. and Taylor, B.A., Spectral analysis of M/G/1 and G/M/1 type Markov chains, Adv. Appl. Prob. 28 (1996), 114-165.

[4] Gross, D. and Harris, C.M., Fundamentals of Queueing Theory, 2nd ed., John Wiley and Sons, New York 1985.

[5] Heffes H. and Lucantoni, D.M., A Markov modulated characterization of packetized voice and data traffic and related statistical multiplexer performance, IEEE J. on Selected Areas in Commun. Vol. SAC-4:6 (Sept. 1986), 856-868.

[6] Latouche G. and Ramaswami, V., Introduction to Matrix Analytic Methods in Stochastic Modeling, SIAM-ASA Series on Stats. and Appl. Prob. 1999.

[7] Lucantoni, D.M., The BMAP/G/1 queue: a tutorial, Models and Tech. for Perf. Eval. of Comp. and Commun. Sys. (ed. by L. Donatiello and R. Nelson), Springer Verlag (1993), $330-358$.

[8] Lucantoni, D.M., Meier-Hellstern, K.S. and Neuts, M.F., New results on the single server queue with a batch Markovian arrival process, Stochastic Models 7:1 (1991), 1-46.

[9] Neuts, M.F., Matrix-Geometric Solutions in Stochastic Models, The Johns Hopkins University Press, Baltimore and London 1981.

[10] Neuts, M.F., Structured Stochastic Matrices of $M / G / 1$ Type and Their Applications, Marcel Dekker Inc. 1989.

[11] Neuts, M.F., A versatile Markovian point process, J. Appl. Prob. 16 (1979), 764-779.

[12] Nishimura, S., Eigenvalue expression for mean queue length of BMAP/G/1 queue, AsiaPacific J. of Opnl. Res. 15 (1998), 193-202.

[13] Nishimura, S. and Jiang, Y., Spectral analysis of the matrix generating function for an MAP/SM/1 queue, Stochastic Models 16:1 (2000), 99-120.

[14] Nishimura, S. and Sato, H., Eigenvalue expression for a batch Markovian arrival process, J. O.R. Soc. Japan 40:1 (1997), 122-132.

[15] Ramaswami, V., The N/G/1 queue and its detailed analysis, Adv. Appl. Prob. 12 (1980), $222-261$. 
[16] Takagi, H., Queueing Analysis, Volume 1: A Foundation of Performance Evaluation, Vacation and Priority Systems, North-Holland, Amsterdam 1991.

[17] Yousef, S.Y. and Schormans, J.A., Performance, interarrival, and correlation analysis of four-phase MMPP model in ATM-based B-ISDN, IEEE Proc. Commun. 143:6 (1996), 363-369. 


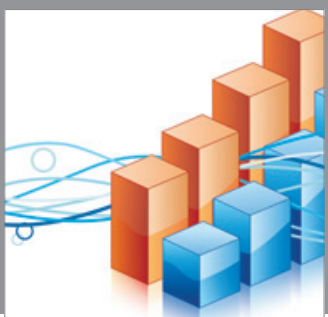

Advances in

Operations Research

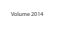

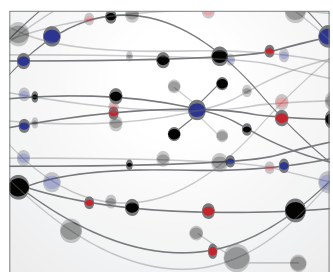

\section{The Scientific} World Journal
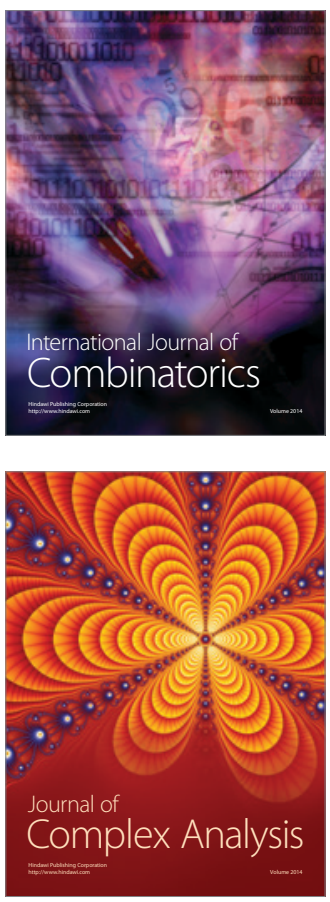

International Journal of

Mathematics and

Mathematical

Sciences
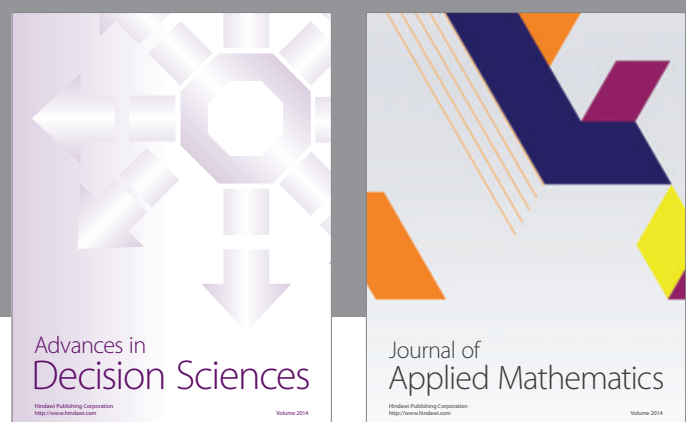

Journal of

Applied Mathematics
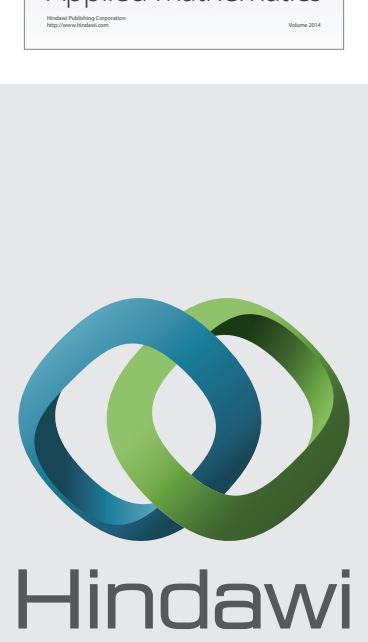

Submit your manuscripts at http://www.hindawi.com
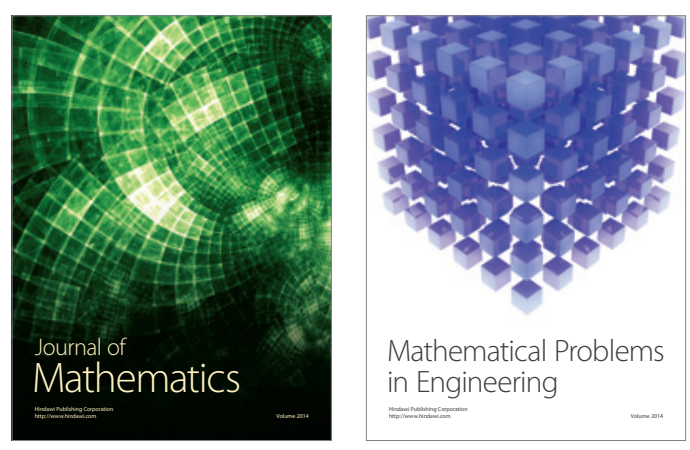

Mathematical Problems in Engineering
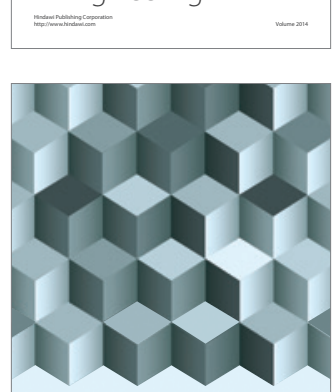

Journal of

Function Spaces
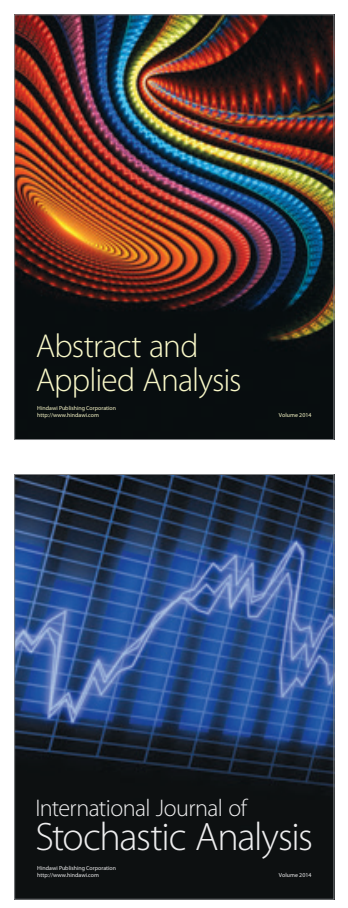

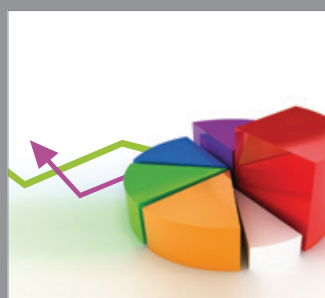

ournal of

Probability and Statistics

Promensencen
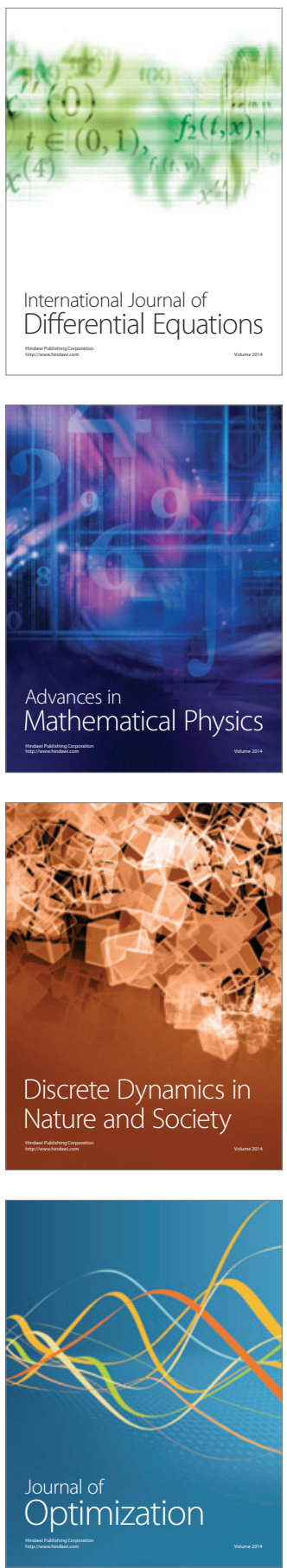